\title{
La longévité du pollen de colza
}

\section{Pollen longevity of oil seed rape}

Oléagineux, Corps Gras, Lipides. Volume 9, Numéro 1, 11-3, Janvier - Février 2002, La filière

Auteur(s) : Jacqueline PIERRE, Michel RENARD, UMR Inra/Ensar, BiO3P, BP 35327, 35653 Le Rheu Cedex.

Résumé : Connaître la longévité d'un pollen est une donnée particulièrement intéressante dans le cadre des études de risques de dissémination du pollen. En effet, cela permet de savoir combien de temps une parcelle dont la floraison est terminée reste une source de pollen fécondant. Par ailleurs, une étude de la longévité du pollen pourrait permettre de mieux comprendre les résultats en apparence contradictoires obtenus au cours de diverses expérimentations menées sur la dissémination du pollen de colza. Ainsi, alors que des travaux montrent que ce pollen est peu anémophile [1] et qu'au niveau de la parcelle sa dispersion se fait sur de courtes distances [2-4], des cas de contaminations sur de grandes distances ont été signalés [5] et les relevés palynologiques effectués sur des filtres (placés à $10 \mathrm{~m}$ de hauteur, voire plus) confirment que ce pollen peut se disperser sur de grandes distances [6]. Toutefois, on ignore si le pollen ainsi collecté est viable ou non. Si l'on fait l'hypothèse que cette dernière dispersion se fait par le biais de la mise en suspension du pollen dans l'air dans les couches de l'atmosphère et que son transport dure un certain temps, il devient également nécessaire de connaître la durée de vie du pollen pour mieux évaluer les risques de ce type de dissémination. Au plan méthodologique, mesurer la longévité du pollen peut a priori paraître simple. Cependant les critères retenus peuvent être nombreux et divers : mesure de la viabilité, mesure du pouvoir germinatif in vitro ou in vivo, mesure du pouvoir fécondant réel. Dans chacun des cas, les techniques appliquées sont différentes et chacune comporte des biais parfois importants tels que des faux-positifs dans les tests colorimétriques de viabilité, l'incidence du milieu de culture dans les tests de germination in vitro, sans parler des tests présentant des risques carcinogènes pour l'expérimentateur [7]. De plus, certains tests ne donnent que des résultats d'ordre qualitatif et l'erreur majeure réside le plus souvent dans une sous-estimation de la longévité. L'objectif de notre travail a donc été double : d'une part, mesurer la longévité du pollen de colza dans diverses conditions de température et d'hygrométrie correspondant à des situations type et d'autre part, tester cette longévité selon deux méthodes très contrastées afin de valider au mieux les résultats et choisir à l'avenir la méthode la plus rapide et la plus fiable. Du pollen de colza (cv. Tanto) a été prélevé au tout début de l'anthèse sur des plantes cultivées au champ. Le pollen a été maintenu durant 15 jours dans les quatre conditions de température et d'humidité relatives suivantes : traitement 1 : conditions extérieures du 9 au 31 juillet 2001, $\mathrm{T}^{\circ}$ de 11 à $18{ }^{\circ} \mathrm{C}, \mathrm{HR}$ de 66 à $92 \%$; traitement $2: \mathrm{T}^{\circ} 20^{\circ} \mathrm{C}$, HR $40 \%$ (16 h/jour ; $8 \mathrm{~h}$ nuit) ; - traitement $3: \mathrm{T}^{\circ} 20^{\circ} \mathrm{C}$, HR $60 \%$ (16 h/jour ; 8 h/nuit) ; - traitement $4: \mathrm{T}^{\circ} 3$ à $5{ }^{\circ} \mathrm{C}, \mathrm{HR}$ de 40 à $95 \%$ (16 h/jour ; $8 \mathrm{~h} /$ nuit). Les tests ont été effectués sur le pollen aux jours J, soit à J0 (pollen frais), J3, J8, J10, J15. 
Summary : Pollen longevity of oilseed rape was studied under four conditions of temperature and relative humidity (external conditions; $\mathrm{T}^{\circ} 20^{\circ} \mathrm{C}, \mathrm{RH} 40 \% ; \mathrm{T}^{\circ} 20^{\circ} \mathrm{C}, \mathrm{RH} 60 \% ; \mathrm{T}^{\circ} 3$ to $5^{\circ} \mathrm{C}, \mathrm{RH} 40$ to $95 \%$ ) and during 0 to 15 days. Longevity was measured by viability (Triphenyl Tretrazolium Chlorid stainability test) and by pollination effectiveness which was tested on cut female flowers (fruit set and number of seeds per pod). The results showed that the viability decreased until eight days. Low temperature with variable $\mathrm{RH}$ was the most conservative treatment ( $9 \%$ on the 8 th day). In all situations, viability did not exceed 15 days. Testing the effectiveness of pollination was more difficult to manage, but the results were in accordance with the viability tests. Our results indicate a higher longevity than that previously described in the literature (three days). These results lead to two conclusions. First, the TTC stainability test is a rapid and reliable method to study pollen longevity and could be used to study the variability of pollen longevity in various oilseed rape varieties. Secondly, when considering the longevity of pollen under variable conditions and especially under low temperature, it can be assumed that a long distance and efficient pollen dispersal over several days in the atmosphere is possible.

Keywords : oilseed rape, pollen, longevity, dispersal.

\section{ARTICLE}

\section{Mesure de la viabilité par test colorimétrique TTC}

Parmi six tests de viabilité référencés [7], le test de coloration par TTC (Triphényl Tétrazolium Chloride) a été retenu comme paraissant, selon la littérature, le plus fiable et le plus rapide. II est fondé sur une réaction d'oxydo-réduction. Les grains vivants possèdent des enzymes d'oxydoréduction qui participent à la respiration et dont la présence peut être détectée par une réaction de coloration. Dans le cas du test TTC, les grains deviennent rouges alors que les grains morts dont les enzymes ne sont plus fonctionnels restent jaunes. Après une préparation qui prend environ 3 heures, suivie d'une lecture au microscope, le taux de viabilité est directement évalué par le ratio grains colorés en rouge/grains totaux, exprimé en pourcentage. Les comptages ont été faits sur 30 grains et répétés 12 fois par traitement (quatre répétitions de chacune des séries). Une analyse de variance a été faite afin de comparer les traitements entre eux, respectivement aux jours J0 (pollen frais), J3, J5, J8, J15.

Le suivi de l'évolution du taux de viabilité du pollen (figure 1) montre en premier lieu que le pollen frais recueilli pour les tests était de bonne qualité (taux moyen de viabilité de 93,7 \%). Une différence entre les traitements apparaît dès le troisième jour : en particulier le taux de viabilité du traitement en conditions naturelles ( $\mathrm{T} 1=59,9 \%$ ) est très inférieur à celui du traitement en conditions les plus froides ( $\mathrm{T} 4=89,9 \%$ ), les traitements $\mathrm{T} 2$ et $\mathrm{T} 3$ présentent des résultats intermédiaires entre les deux situations (respectivement 77,9 et 85,3\%). À huit jours, les traitements T1, T2 et T3 sont encore significativement différents entre eux mais assez peu comparés à T4. Au bout de 10 jours, seul le traitement T4 permet une assez bonne conservation de la viabilité $(34,5 \%)$ alors que pour tous les autres traitements le taux n'est que de 3,5\% en moyenne. 
Au final, le traitement dans les conditions les plus froides et avec un hygrométrie très variable est le plus conservateur, et le maintien à une température constante de $20{ }^{\circ} \mathrm{C}(\mathrm{T} 2, \mathrm{~T} 3)$ permet une meilleure conservation qu'à des températures et hygrométries très variables (T1). Quel que soit le traitement, la longévité du pollen de colza n'excède pas 15 jours.

\section{Mesure du pouvoir fécondant sur fleurs mâle-stériles coupées}

Afin de tester le pouvoir fécondant du pollen in vivo, le pollen a été déposé sur des fleurs mâlestériles (lignée Fu-Tanto) cultivées au champ, coupées puis maintenues en culture durant 40 jours selon une technique développée par ailleurs $[8,9]$ en veillant au choix des fleurs femelles (stade et position dans la plante). Les dépôts ont été faits de manière à optimiser la pollinisation (stigmate largement recouvert de grains de pollen). Le pouvoir fécondant a été mesuré par le taux de nouaison (nombre de fleurs ayant donné des siliques/nombre de fleurs, en pourcentage) et par le nombre de graines par silique. Soixante fleurs ont été testées par traitement.

Les données relatives au taux de nouaison se sont révélées plus délicates à interpréter que les taux de viabilité. En effet, une fleur non fécondée ne développe pas de siliques à proprement parler et cette silique, de taille très réduite, est très sensible aux attaques par les champignons et bactéries malgré les conditions stériles dans lesquelles sont réalisées les manipulations. Lorsque la silique est contaminée, il est impossible de savoir s'il y eu ou non formation effective d'une silique avec production de graines. Les taux de nouaison sont donc aussi le reflet du taux de survie. Par ailleurs, les comptages de graines par silique bien formée montrent que ceux-ci ne dépassent jamais 9, ce qui est l'indice d'une mauvaise fécondation (espérance habituelle : 20 graines par silique). Cet échec de pollinisation n'atteste pas pour autant une absence de viabilité du pollen mais peut aussi provenir du fait que la plante femelle est placée dans des conditions de survie.

Malgré ces restrictions, il est possible de dégager les conclusions suivantes de l'ensemble des données (figures 2 et 3 ) : le taux de nouaison-survie des plantes pollinisées avec du pollen frais est assez élevé ( $\mathrm{JO}=85 \%$ ) mais le nombre de graines par silique est faible (4 en moyenne, maximum 9). Le taux de nouaison reste non négligeable pour un pollen âgé de huit jours dans le cas des traitement $\mathrm{T} 1, \mathrm{~T} 2, \mathrm{~T} 3$ (J8 de 60 à $77 \%$ ) et il faut noter que c'est une fois encore le traitement T4 qui est le plus conservateur ( $85 \%)$. Entre $\mathrm{J} 0$ et $\mathrm{J} 8$, on assiste à une baisse du taux de nouaison pour tous les traitements, qui est imputable à une contamination par des champignons et des bactéries durant cette période. Au-delà de $\mathrm{J} 8$, le taux décroît très rapidement et devient nul pour un pollen âgé de 15 jours. Le nombre de graines par silique formée décroît de la même manière mais aucune différence n'apparaît entre les traitements sans doute parce que le nombre de graines produites est trop faible.

\section{CONCLUSION}

II s'avère que les deux méthodes appliquées pour étudier la longévité du pollen, bien que très différentes, donnent des résultats concordants. II semble acquis que, pour la variété. "Tanto ", la durée de vie du pollen n'excède pas 10 à 15 jours. Techniquement, l'étude du pouvoir fécondant in vivo sur fleur coupée est longue à mettre en œuvre et comporte de nombreux aléas liés à la technique elle-même. Elle présente l'inconvénient d'introduire une interaction entre le pollen et l'état physiologique de la plante réceptrice, phénomène certes incontournable lorsque l'on effectue 
des test in vivo mais qui, dans ce cas, est accentué par le fait que la fleur femelle est maintenue sur milieu artificiel (risque de contamination). II faut souligner qu'une expérimentation préliminaire portant sur la viabilité du pollen de 0 à 30 jours, dans les mêmes conditions de traitement, avait déjà montré que le pollen avait une durée de vie maximale de 15 jours. Ainsi, à l'avenir, on pourra retenir le test de viabilité par coloration TTC comme étant une technique rapide et fiable pour mesurer la variabilité de la longévité de diverses variétés de colza.

Les résultats montrent que, contrairement à ce qui est généralement rapporté sur les conditions de stockage du pollen $[10,11]$, une hygrométrie élevée n'entraîne pas chez le colza une réduction de la durée de vie, sous réserve que les températures soient assez basses ( 3 à $5{ }^{\circ} \mathrm{C}$ ). De même les fortes pluies et fortes variations de température ou d'ensoleillement enregistrées en conditions extérieures (bilan sur 2 années) permettent une durée de vie d'une semaine à 10 jours. Ces valeurs sont supérieures à celles trouvées au cours de tests de germination effectués in vitro (technique de la goutte pendante) lors d'études précédentes et qui étaient de 3 jours [12].

Au vu de nos derniers résultats, on peut admettre qu'une parcelle source restera contaminante un peu plus d'une semaine voire 15 jours après la fin de floraison, même si ce risque reste limité compte tenu de la diminution progressive de la quantité de pollen émise en fin de floraison. Par ailleurs, en ce qui concerne les contaminations à distance au cours de la pleine floraison, on peut admettre que le pollen, quand il est mis en suspension dans les couches moyennes de l'atmosphère (abaissement de la température de $0,7{ }^{\circ} \mathrm{C}$ par élévation de $100 \mathrm{~m}$ ) conserve une viabilité qui permettra des contaminations même après plusieurs jours de transport par le vent dans des conditions d'hygrométrie très variables.

\section{Remerciements}

Ce travail a été réalisé avec l'appui technique d'Arnaud Le Pors. Il a été effectué dans le cadre de I'Action incitative programmée de I'Inra « OGM et Environnement ».

\section{REFERENCES}

1. EISIKOWITCH D (1981). Some aspects of pollination of oilseed rape. J agric Sci Cambridge, 96 : $321-$ 6.

2. LAVIGNE C, KLEIN EK, VALLEe P, PIERRE J, GODELLE B, RENARD M (1998). A pollen dispersal experiment with transgenic oilseed rape. Estimation of the average pollen dispersal of an individual plant within a field. Theor Appl Gen, $96: 886-96$.

3. MESQUIDA J, RENARD M (1982). Étude de la dispersion du pollen par le vent et de l'importance de la pollinisation anémophile chez le colza. Apidologie, 13 : 353-66.

4. MESQUIDA J, RENARD M (1984). Étude des quantités de pollen déposées sur les stigmates dans différentes conditions de pollinisation, influence sur la production de graines chez les colzas d'hiver mâle-fertiles. In : Cinquième symposium sur la pollinisation, Inra ed., Les colloques de I'Inra, 21 : 3516.

5. TIMMONS AM, O'BRIEN ET, CHARTERS YM, DUBBELS SJ, WILKINSON MJ (1995). Assessing risk of wind pollination from field of genetically modified Brassica napus ssp oleifera. Euphytica, 85 : 417-23. 
6. MCCARTNEY HA, LACEY ME (1991). Wind dispersal of pollen from crops of oilseed rape (Brassica napus). J Aerosol Sci, 22 : 467-77.

7. DAFNI D (1992). Pollination ecology, a practical approach. Oxford : Oxford University Press, 250 p.

8. LARDON A (1991). Mise au point d'une technique de culture de fleurs isolées chez le colza. Rapport de stage, Univ. Claude-Bernard, Lyon.

9. TRIBOI-BLONDEL AM, CASTANO-COLABELLI M, MERRIEN A (1991). Germinability and viability of rapeseed pollen under the effect of temperature. Proc 8th International Rapeseed Congress, Saskatoon, Canada, 9-11 July $1991 ; 6$ : 1758-63.

10. Hanna WW, Towill LE (1995). Long-term pollen storage. Plant Breeding Rev, 13 : 179-207.

11. STANLEY RG, LINSKENS HF (1974). Pollen: biology, biochemistry, management. Berlin, Heidelberg, New York : Springer-Verlag, $307 \mathrm{p}$.

12. MESQUIDA J, RENARD M, MESQUIDA B (1987). Étude préliminaire sur la germination in vitro du pollen de colza (Brassica napus L. var. oleifera Metzg.) et sur l'évolution dans le temps de son aptitude à germer. Agronomie, $7:$ 409-16.

Illustrations

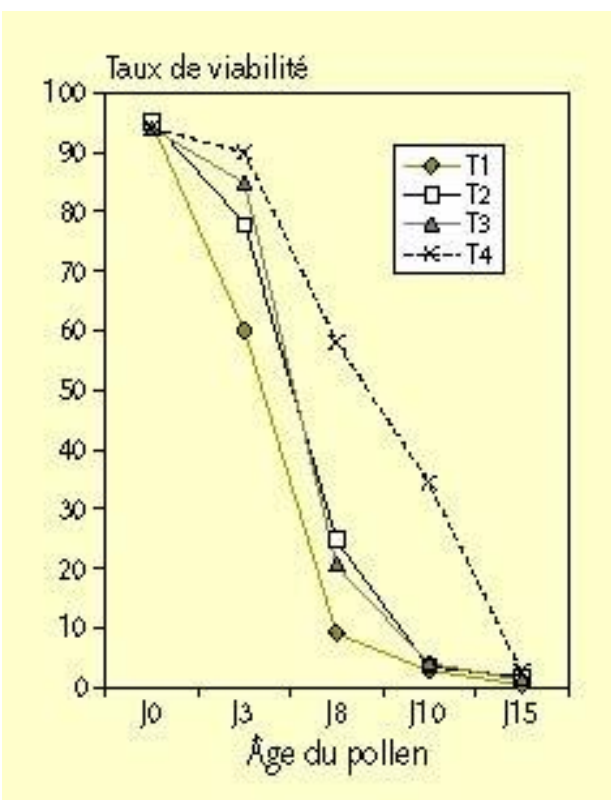

Figure 1. Évolution du taux de viabilité du pollen de colza (Test TTC) soumis à quatre traitements. $T 1$ : conditions extérieures; $T 2: 20{ }^{\circ} \mathrm{C}, H R 40 \% ; T 3: 20{ }^{\circ} \mathrm{C}, H R$ $65 \%$; T4 : 3 à $5{ }^{\circ} \mathrm{C}$, HR 40 à $95 \%$. 


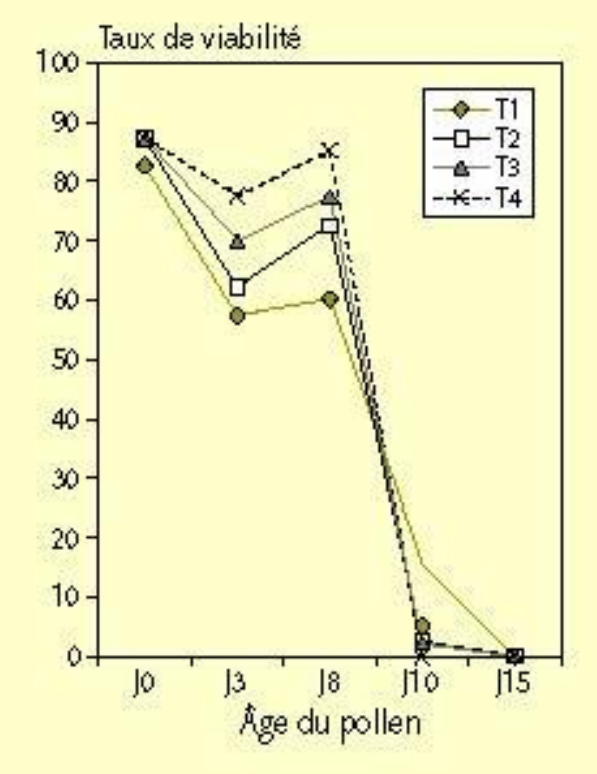

Figure 2. Évolution du taux de nouaison après fécondation de fleurs mâle-stériles par du pollen de colza soumis à quatre traitements. $T 1$ : conditions-extérieures ; T2 : $20{ }^{\circ} \mathrm{C}, \mathrm{HR} 40 \%$; T3 : $20{ }^{\circ} \mathrm{C}, \mathrm{HR} 65 \%$; T4 : 3 à $5{ }^{\circ} \mathrm{C}, \mathrm{HR} 40$ à $95 \%$.

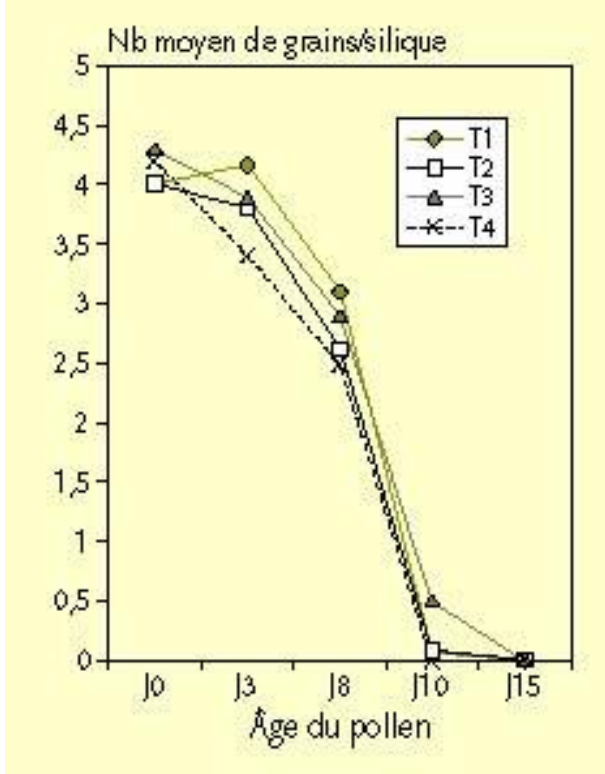

Figure 3. Évolution du nombre de graines produites par silique après fécondation de fleurs mâle-stériles par du pollen de colza soumis à quatre traitements. $T 1$ : conditions extérieures; $T 2: 20{ }^{\circ} \mathrm{C}, \mathrm{HR} 40 \% ; \mathrm{T3}: 20^{\circ} \mathrm{C}, \mathrm{HR} 65 \%$; T4: 3 à $5{ }^{\circ} \mathrm{C}, \mathrm{HR}$ 40 à $95 \%$. 
Photo 1. Pollen de Colza au MEB. Pollen dans I'anthère déhiscente.

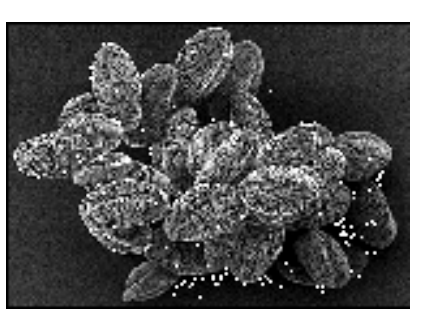

Photo 2. Pollen de Colza au MEB. Amas de grains de pollen.

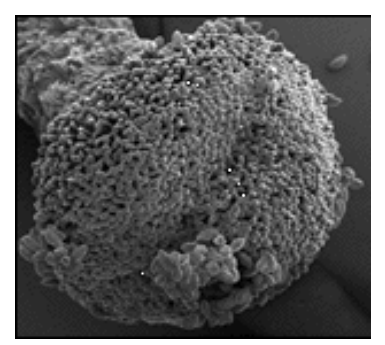

Photo 3. Dépôt de pollen de colza sur le stigmate. Vu au MEB.

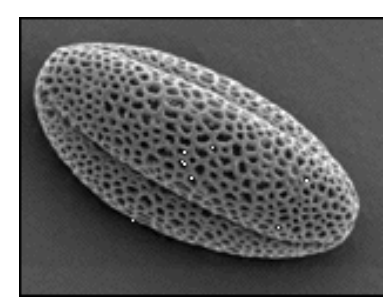

Photo 4. Grain de pollen de colza au MEB. Préparation directe (longueur 30 mu). 